\title{
Structural Transitions of Silicate Nanocrystals in the Glass
}

\author{
G. Abdurakhmanov*, G. S. Vakhidova
}

Institute of Power Engineering and Automation, the Uzbek Academy of Sciences, 29 Do'rmon yo'li, Tashkent, 100125 Uzbekistan

\begin{abstract}
The temperature dependences of resistivity and thermopower measurements reveal that nanocrystals of silicates in lead-silicate glass of various compositions undergo structural transitions in the temperature range of $800-1000 \mathrm{~K}$. The diameter of these nanocrystals estimated from Scherrer's formula is about $0.8-1.6 \mathrm{~nm}$ and each nanocrystal consists of 8 - 64 unit cells. Structure transitions are detected as sharply maxima of resistivity $\rho$ and thermopower $\mathrm{S}$ at temperature $\mathrm{T}=800-100 \mathrm{~K}$. Lead-silicate glass was doped by $\mathrm{RuO}_{2}$ up to 16 weight $\%$ to facilitate the measurements of the $\rho$ and the $\mathrm{S}$. The doped lead-silicate glass has a metal-like behavior at the room temperature: the temperature dependences $\rho(\mathrm{T})$ and $\mathrm{S}(\mathrm{T})$ are very slow, the value of the $\mathrm{S}$ is typical for metals (few and tens of $\mu \mathrm{V} / \mathrm{K}$ ). Beyond the maximum of the resistance (temperature $\mathrm{T}>1000 \mathrm{~K}$ ) doped lead-silicate glass turns into typical semiconductor having energy gap about $0.05-1.5 \mathrm{eV}$ depending on the composition of the glass. Anomalous thermal expansion of the $\mathrm{RuO}_{2}$ relict crystals is detected at the temperatures of $800-1000 \mathrm{~K}$ as well.
\end{abstract}

Keywords structure transitions in nanocrystals, silicate glass, thermopower, resistivity, doping, thermal expansion

\section{Introduction}

Structural transitions as a fundamental property of interacting atoms in the crystalline state are very important for basic knowledge and applications. These transitions are collective phenomenon and therefore should depend on the number of atoms in the sample, i.e. on the size of the latter one. For example, the Curie temperature $T_{\mathrm{C}}$ for nanostructured ferroelectrics decreases with the size $d$ of nanocrystals as $d^{-1}$, with it's lowest value reaching $T_{\mathrm{c}} \approx 300 \mathrm{~K}$ at $d \approx 3$ $\mathrm{nm}$ (fig. 3 in [1]). The size of nanocrystals in these investigations was defined as the diameter of nanowire, i.e. in two directions only, while in the third direction (along the wire) the value of this parameter might be significantly higher.

Structural transitions in nanocrystals of $\mathrm{LaMnO}_{3}$, having average dimensions about $15-20 \mathrm{~nm}$, were investigated in [2]. It was found that transition temperature $T_{\mathrm{C}}$ in these nanocrystals undergoes a reduction up to $T_{\mathrm{C}}=493 \pm 10 \mathrm{~K}$ in compared to $T_{\mathrm{C}}=750 \mathrm{~K}$ in the bulk samples.

To our knowledge, there is no information about structural transitions in smaller nanocrystals and in the nanocrystals formed in the glass.

All the silicate glasses have been devitrificated in time (tens or hundreds of years) so one can assume that nanocrystals of silica and various silicates are formed naturally

* Corresponding author:

gulmirzo@mail.ru (G. Abdurakhmanov)

Published online at http://journal.sapub.org/materials

Copyright (C) 2012 Scientific \& Academic Publishing. All Rights Reserved in silicate glasses and structural transformations should take place in them. Dimensions of these nanocrystals are $1-2$ $\mathrm{nm}$ as follows from X-ray diffraction patterns (Scherrer's formula). For example, the structural transition of $\beta$-quartz into $\alpha$-quartz and vice versa takes place at $846 \mathrm{~K}$ and is accompanied by the increase of the unit cell volume by about $6 \%[3]$. Increase of interatomic distances due to the structural transitions leads to decrease in the electrical conductivity of the sample, so this effect can be exploited for collecting the information about the structural transitions. The unit cell of the $\beta$-quartz is trigonal with $a=0.4893 \mathrm{~nm}, c=$ $0.5384 \mathrm{~nm}$ at the room temperature, while the $\alpha$-quartz is hexagonal with $a=0.4983 \mathrm{~nm}, c=0.5419 \mathrm{~nm}$ at $848 \mathrm{~K}$.

Direct and doubtless evidences of existence of nanocrystals in the glass have not been thoroughly investigated before. That is why the problem of the glass structure (continuous random network, Zachariasen, 1932; microcrystallits, separated by interlayer of disordered atoms, Lebedev, 1921) is still the subject of discussions. But the temperature dependence of some glass properties such as viscosity, thermal expansion coefficient, dielectric constant and dielectric loss, have anomalies which have a better agreement with microcrystalline theory. This is especially the case of manycomponent glasses.

Here we show that structural transitions take place in nanocrystals of the average size of $1-2 \mathrm{~nm}$ in doped lead-silicate glass and are accompanied by sharp increase of the resistivity $\rho$ and the Seebeck coefficient $S$ at the temperatures below $1123 \mathrm{~K}$. We found that at these temperatures the value of $\rho$ is $2-6$ times higher than that value at room 
temperature and $S$ may be as high as $10^{3} \mu \mathrm{V} / \mathrm{K}$. Both $\rho$ and $S$ are affected by chemical composition of the glass.

Direct investigations of the structure of nanocrystals smaller than $3-4 \mathrm{~nm}$ by diffraction methods (X-ray or neutron) are problematic, so one is forced to probe them by the indirect methods such as the measurements of the electrical properties (conductivity and thermopower). For example, structure transformations in ferroelectrics leads to sharp increase of resistivity $\rho$ and decrease of permittivity $\varepsilon$ as the interatomic distances increase[4].

\section{Experiment}

The samples of glass were doped by $\mathrm{RuO}_{2}$ to reduce the resistivity and to enhance the thermopower in our experiments, so it is possible to measure the reduced value of resistivity of about $10^{-2}-10^{5} \mathrm{Ohm} \cdot \mathrm{cm}$ more accurately than that of the glass (about $10^{15}-10^{16} \mathrm{Ohm} \cdot \mathrm{cm}$ ). The doping process used here is standard for technology of thick film resistors and was described elsewhere[5] (the mixture of the glass and the dopant powders have been fired at $T_{\mathrm{f}}=1125$ $\mathrm{K}$ in $\tau=10 \mathrm{~min}$ ). Content of $\mathrm{RuO}_{2}$ in our samples of doped glass was 16,20 and 30 weight $\%$. The lead-silicate glasses investigated have the following composition (weight \%):

Glass $1 \quad \mathrm{SiO}_{2}-33 ; \mathrm{PbO}-67$;

Glass2 $\mathrm{SiO} 2-27 ; \mathrm{PbO}-67 ; \mathrm{BaO}-4 ; \mathrm{MgO}-2$.

The mixture of oxides have been melted at temperatures $1723 \pm 20 \mathrm{~K}$ (glass1) and 1623 $\pm 20 \mathrm{~K}$ (glass2) for $1 \mathrm{~h}$. Density of the glasses by hydrostatic weighing is $4.81 \pm 0.02$ $\mathrm{g} \cdot \mathrm{cm}^{-3}$ for the glass 1 and $5.13 \pm 0.02 \mathrm{~g} \cdot \mathrm{cm}^{-3}$ for the glass 2 . The glass transition temperature $T_{\mathrm{g}}$ have been evaluated by thermomechanical analysis (TMA, temperature dependence of the thermal expansion coefficient) and was found to be $760 \pm 20 \mathrm{~K}$ for the glass 1 and $785 \pm 20 \mathrm{~K}$ for the glass 2 .

Samples of the doped glass for electrical measurements were the bulk material: $5 \mathrm{~mm}$ in diameter and $10 \mathrm{~mm}$ in height with the Pt or Ag electrodes. Samples for X-ray investigations were powders. The Pt heater of the D500 Siemens X-ray diffractometer was coated by these powders in the nitrocellulose lacquer. In the final X-ray patterns the diffraction lines of Pt have been deleted.

The values of resistance and thermopower were measured by digital multimeter Sch-300 having error less than $0.2 \%$. The temperature was measured by the same multimeter and standard Pt-PtRh thermocouple. The thermocouple was calibrated in standard temperature points of water boiling $(373 \mathrm{~K})$, tin and silver solidification (505 and $1234 \mathrm{~K}$ accordingly).

Accuracy of determining of the lattice parameters of $\mathrm{RuO}_{2}$ was about $\pm 0.01 \AA$ and estimated from Bregg's formula[6] for interplane distance $d=\lambda / 2 \sin \theta$ as

$$
\Delta d / d=\Delta \lambda / \lambda+\Delta \theta \cos \theta / \sin \theta,
$$

here wavelength $\lambda=0.15418 \pm 0.00001 \mathrm{~nm}$ for $\mathrm{CuK \alpha}$ radiation, $\theta$ is the diffraction angle, $\Delta d, \Delta \lambda$ and $\Delta \theta$ are absolute errors of corresponding values. The crystallographic planes [002] and [220] of $\mathrm{RuO}_{2}$ (rutile structure, tetragonal system) appear at $2 \theta=59.5$ and 58.1 accordingly in our experiments. The accuracy of the $2 \theta$ is about 0.05 for Siemens D500 diffractometer so $d_{[002]}=1.554 \pm 0.002 \AA$ and $d_{[220]}=1.588 \pm 0.002 \AA$. The according values of the lattice parameters are $c=2 d_{[002]}=3.108 \pm 0.004 \AA$ and $a=2 \sqrt{2} d_{[220]}$ $=3.176 \pm 0.006 \AA$.

The mean size $L$ of the nanocrystals in the glass was estimated for the main halo at about $2 \theta \approx 30-35^{\circ}$ via Scherrer's formula[6] $L=K \lambda /(B \cos \theta)$, here $K$ is the dimensionless shape factor (about 0.9 ) and $B$ is the line broadening at half the maximum intensity (in radians, see fig. 1). Accuracy of the $L$, estimated without standards, is essentially worse (> $10 \%$ ) than that of the interatomic distances (see above) because of the errors in $K, B$ and the instrumental distortions.

We do not use the transmission electron microscopy (TEM) to investigate the nanocrystals in the silicate glass for the following reasons: i) very thin films of the material are required to investigate by TEM, and preparation process of them can significantly affect the structure of nanocrystals in a glass; ii) these thin films of the silicate glass loose the shape due to firing up to $700-800 \mathrm{~K}$ and form drops, so TEM investigations become impossible.

\section{Results and Discussion}

The X-ray diffraction patterns for glass 1 before and after doping are shown in fig. 1. From Scherrer's formula for $\mathrm{CuK} \alpha$ radiation $(\lambda=0.15418 \mathrm{~nm})$ we evaluate the size of nanocrystals $L \approx 0.87 \mathrm{~nm}$ in the undoped glass1 and $L \approx$ $1.53 \mathrm{~nm}$ in the doped glass1. The same values were found for the glass 2 as well. The main reflexes of polymorphs of silica and the lead silicates are in area $d=3-5 \AA$. This conforms to wide halo at $2 \theta \approx 31.86^{\circ}$ for nondoped glass 1 (having molecular composition $2 \mathrm{SiO}_{2} \cdot \mathrm{PbO}$ ) (fig.1).

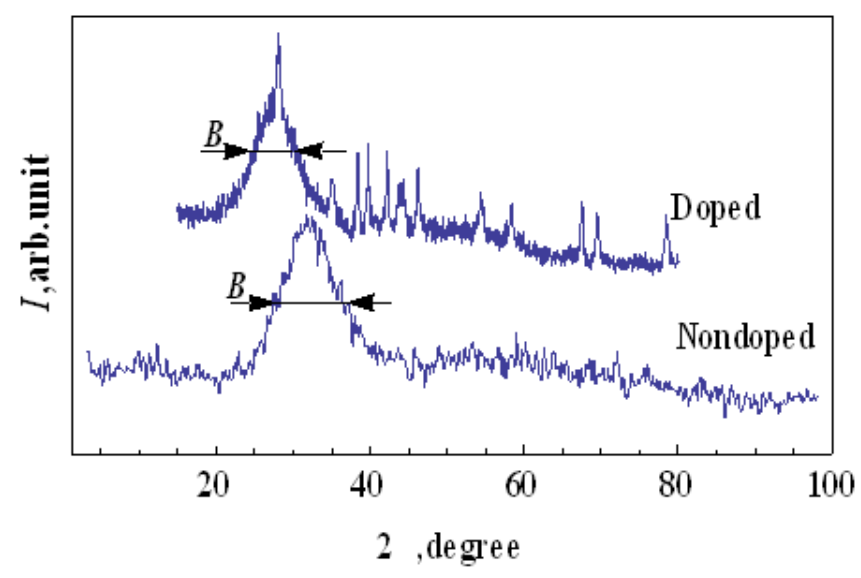

Figure 1. X-ray diffraction pattern for glass1 befor and after doping. Sharp peaks are due to relicts of $\mathrm{RuO}_{2} . B$ is the line broadening at half maximum of intensity (in radians)

Temperature dependences of the resistance $R(T)$ and the Seebeck coefficient $S(T)$ of the doped glass1 and the doped glass2 are shown in fig. 2 and 3 accordingly. Values of $R, S$ and $T$ have been measured with accuracy of about $1 \%$. $R(T)$ and $S(T)$ at $T<800 \mathrm{~K}$ are nearly constant, so one can as- 
sume the free charge carriers concentration $n$ does not change. Sharp increase of the $R(T)$ and $S(T)$ at $T>800-$ $900 \mathrm{~K}$ is the result of the structural transitions of silica and lead-silicate nanocrystals because the relict crystals of $\mathrm{RuO}_{2}$ do not have appropriate variations (fig. 4). This statement follows from the fact that increase of the interatomic distances in solids leads to decrease of its electrical conductivity. In the case of $\mathrm{RuO}_{2}$ crystals investigated, we have the opposite situation - lattice parameters, both $a$ and $c$, decrease in the temperature range $800-1000 \mathrm{~K}$, where resistance $R(T)$ of the sample increases (please compare fig. 2 and 3 with the fig. 4).

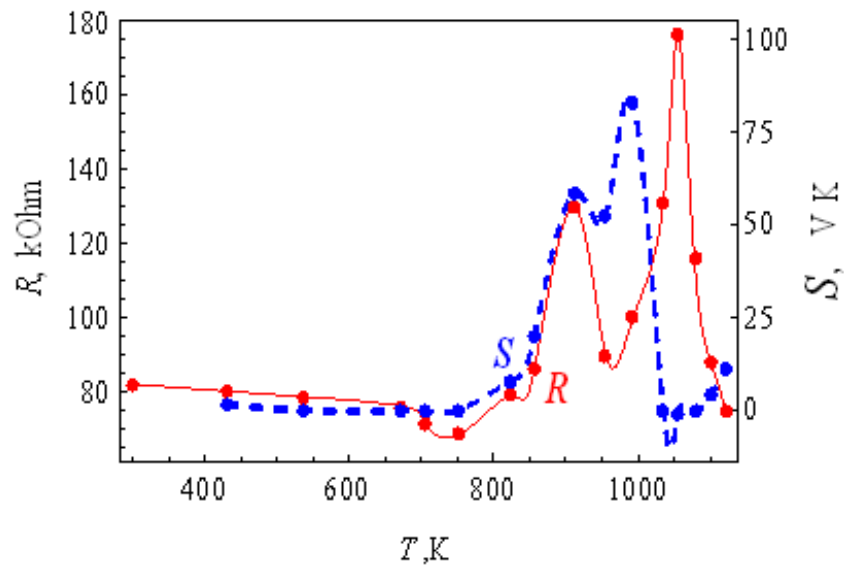

Figure 2. Temperature dependence of the resistance $R(T)$ and the thermopower $S(T)$ for the doped glass 1 .

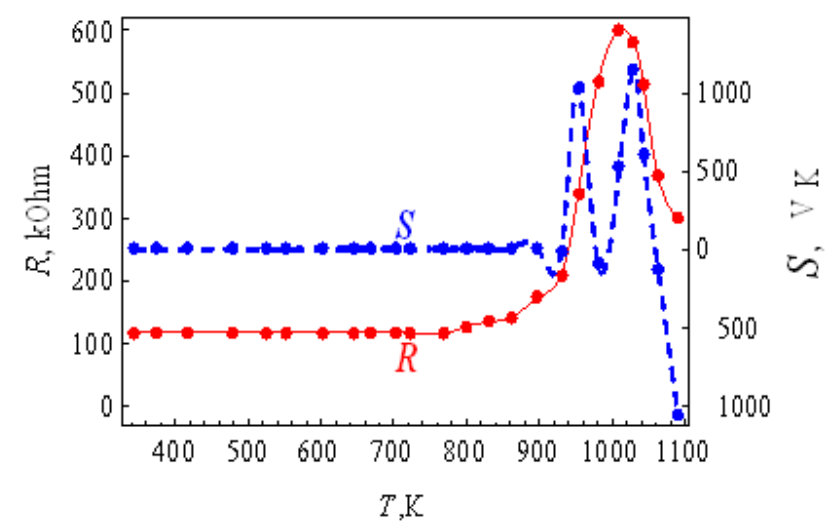

Figure 3. Temperature dependence of the resistance $R(T)$ and the thermopower $S(T)$ for the doped glass 2

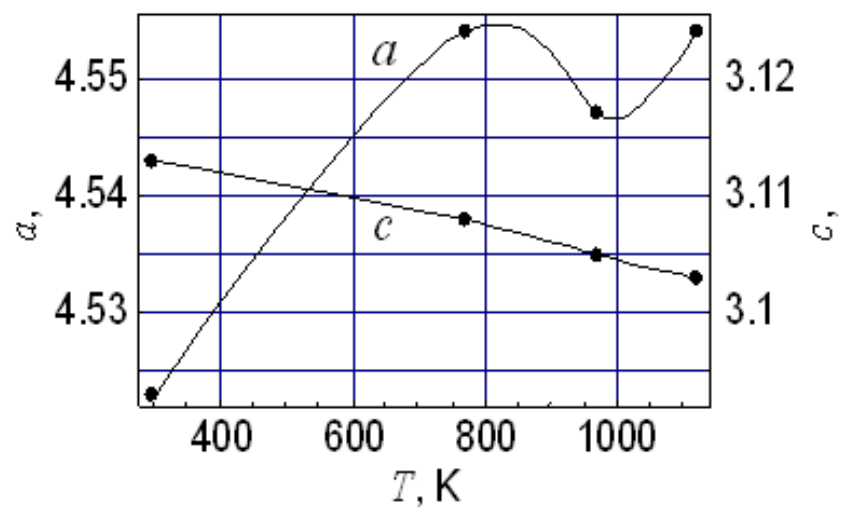

Figure 4. Temperature dependence of the lattice constants of $\mathrm{RuO}_{2}$ crystals in the free powder in $a$ and $c$ directions
It is noteworthy that anomalous behavior of the thermal expansion of $\mathrm{RuO}_{2}$ crystals in $a$ direction takes place (fig. 4) - extension is replaced by constriction in the temperature range $800-1000 \mathrm{~K}$ followed by extension again.

One can see from fig. 2 and 3 that the temperature dependences of $R$ and $S$ are very weak and the value of $S$ is few or ten $\mu \mathrm{V} / \mathrm{K}$ only at $T<800 \mathrm{~K}$ for doped glasses.

After maximum of $R(T)$ the doped glass turns into typical semiconductor having band gap width between 0.05 and 1.5 $\mathrm{eV}$ for various glass compositions and doping levels (fig. 5). This means that the structural transition shifts away the impurity subband from the top of the glass valence band.

The comparison of the unit cell parameters of low- temperature $\beta$-quartz with the size of nanocrystals in the glass shows that the last one consists of $8-60$ unit cells, or $24-$ 180 atoms of $\mathrm{Si}$, enclosed by mostly disordered layer. The effect of this layer on structural transitions of nanocrystals is not clear yet. In the case of the lead-silicates having lager unit cells, the number of the unit cells in the nanocrystals is smaller than that of the quartz.

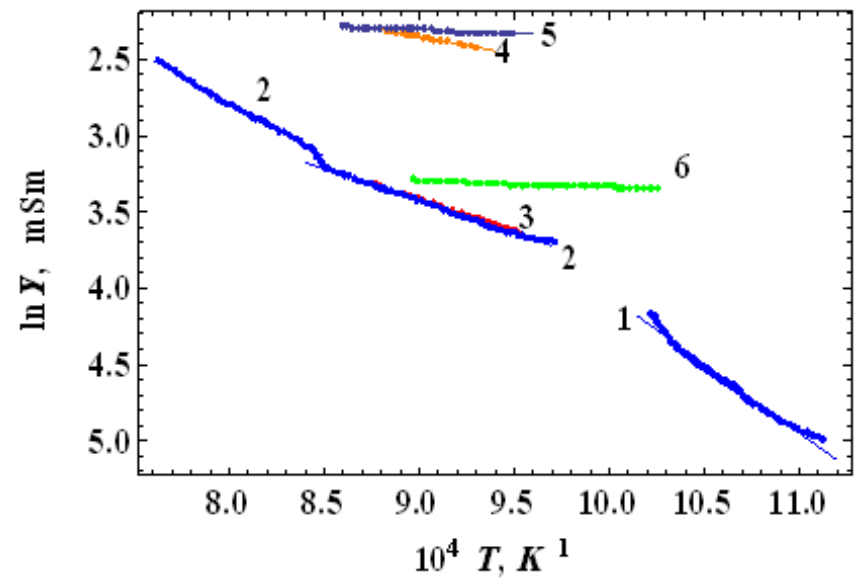

Figure 5. Temperature dependence of the conductivity $Y(T)$ after maximum for the samples of the doped glass1 (1) and glass2 $(2-6)$ with $\mathrm{RuO}_{2}$ content about $16 \mathrm{wt} \%(1,2), 20 \mathrm{wt} \%(3,4)$ and $30 \mathrm{wt} \%(5,6)$. Activation energy of conductivity $E_{\sigma}$ is about $(\mathrm{eV}): 1.77(1) ; 1.54(2$, lower $T)$ and 0.93 (2, high T); 0.9 (3); 0.52 (4); 0.05 (5) and 0.047 (6). The solid lines are the fit of the experimental data with the expression $\ln Y=a+E_{\sigma} /(2 k T)$

\section{Conclusions}

Nanocrystals of silica and silicates with the size of $0.8-$ $1.6 \mathrm{~nm}$ undergo structural transitions at the temperatures $800-1000 \mathrm{~K}$, detected as sharp maxima of the resistance and thermopower of the lead-silicate glass, doped by $\mathrm{RuO}_{2}$. Before these maxima the temperature dependence of the parameters and the value of the thermopower are typical for metals, while after maxima resistance assumes an activation character with activation energy of $0.05-1.5 \mathrm{eV}$ depending on the glass composition. It is shown that the $\mathrm{RuO}_{2}$ relict crystals are not responsible for these anomalies because of the lattice constriction in the temperature region of these maxima $(800-1000 \mathrm{~K})$ and reduction of their resistivity. At maxima the value of the resistance may be $6-10$ times 
higher than that at room temperature, and value of the thermopower is $10-100$ times higher than at room temperature.

\section{ACKNOWLEDGEMENTS}

We acknowledge the Uzbek Academy of Sciences for the financial support (Grant in the Support of Fundamental Research, grant NN 55-08 and 27-10).

\section{REFERENCES}

[1] J. M. Gregg, Ferroelectrics at the nanoscale, Phys. St. Solidi A 206 (2009) 577-587. DOI 10.1002/pssa.200824434
[2] M. V. Harlamova and A. Arulraj, Phase transition in nanostructured $\mathrm{LaMnO}_{3}$, JETP Letters 89 (2009) 301-305.

[3] V. P. Pryanishnikov, Sistema kremnezema (Silica system in Russian). Building Literature Publications. - Leningrad, 1971.

[4] M. E. Lines and A. M. Glass, Principles and applications of ferroelectrics and related materials. Clarendon Press. - Oxford, 1977.

[5] Thick Film Sensors. Ed. M. Prudenziati. Elsevier Science. Amsterdam, 1994.

[6] A. Guinier, Théorie et technique de la radiocristallographie. Dunod. - Paris, 1956.

[7] Vitalij K. Pecharsky, Peter Y. Zavalij, Fundamentals of powder diffraction and structural characterization of materials. Springer. - N. Y., London, 2005. 